\title{
Before-after study comparing systematic labour induction at 41 weeks of amenorrhea and 3 days versus 41 weeks of amenorrhea and 5 days in a maternity ward in France in 2017-2018
}

\author{
Enora PARC*, Zelda STEWART and Estelle WAFO \\ Hospital of the Eastern Paris Hospital Group, site Jossigny, France
}

\begin{abstract}
Objectives: In France, close surveillance is recommended from 41 weeks of amenorrhea (WA) and induction of labor before 42 WA, with no clear consensus on a precise term. Our objective was to compare two systematic induction terms, $41 \mathrm{WA}+3$ days versus $41 \mathrm{WA}+5$.

Method: A retrospective observational before-after study took place in our maternity ward. The first period ran from September to November 2017 with systematic induction at 41WA+3, the second from March to May 2018 with systematic induction at 41WA+5. The primary outcome was a composite criterion of perinatal morbidity.

Results: We included 118 patients. There were less inductions for term only and more inductions for oligoamnios, decreased feeling of active fetal movement, fetal heart rate abnormalities, and premature rupture of the membranes in the after group $(\mathrm{p}<0.01)$. There was no significant difference in the cesarean section rate, color of amniotic fluid, perineal lesions, blood loss. There was also no difference between neonatal characteristics (birth weight, APGAR score, umbilical pH).

Conclusion: In the after population, the caesarean section rate was not increased, the spontaneous labor rate was higher, and the neonatal state similar. The decrease of inductions for term only and the parallel increase of inductions for medical reasons shows that maternal and fetal monitoring has been systematic and rigorous. This study allows additional arguments to induce labor at $41 \mathrm{WA}+5$. It confirmed the implementation of this new protocol in our maternity.
\end{abstract}

\section{Introduction}

In France, the term of pregnancy is considered to be between 37 weeks of amenorrhea (WA) and 41 WA and 6 days [1]. By convention, it is established that pregnancy is said to be out of date from 42 WA. In France in 2016, a frequency of $16.8 \%$ of patients (all populations combined) giving birth between $41 \mathrm{WA}$ and $41 \mathrm{WA}$ and 6 days, against $0.5 \%$ beyond 42 WA [2-3].

Maternal and foetal consequences of prolonged pregnancy and post term pregnancy are now well known. From a maternal point of view, postterm is associated with an increase in caesarean section rate and a moderate increased risk of third- and fourth-degree perineal lesions, postpartum haemorrhage and chorioamnionitis [4-7].

From a fetal point of view, risk of perinatal morbidity and mortality is increased: increased risk of oligoamnios, abnormal fetal heart rate, meconium inhalation [4-7]. Similarly, the risks of neonatal acidosis, low APGAR score, and admission to intensive care are more important. Newborns are more likely to have neurological complications such as neonatal seizures, anoxic ischemic encephalopathy, cerebral palsy. Finally, they have a 5-fold higher risk of macrosomia and associated obstetric complications [7-12].

Therefore, many studies that focus on the subject come to the same conclusion: benefit-risk balance is clearly against a pregnancy that extends beyond 42 weeks. In this context, the French High Authority for Health (HAS) issued recommendations in 2008 and 2011: The HAS recommends a rigorous monitoring from $41 \mathrm{WA}$, every 48 hours. In absence of delivery, it is recommended to perform a labour induction, possibly preceded by a cervical ripening with prostaglandins, so patient give birth before $42 \mathrm{WA}$.

It should be noted that these recommendations also underline need for a first trimester ultrasound for an accurate determination of the term from the measurement of the cranio-caudal length of the fetus. Its systematic practice contributes to reducing frequency of terms that are wrongly considered out of date [13].

However, there is currently no consensus on ideal term of systematic induction between 41 WA and 41 WA and 6 days: What term exactly should be used? How and under what conditions? Is there a difference in maternal and fetal prognosis based on this induction term?

The aim of our study was to compare two systematic induction terms, 41 WA and 3 days versus 41 WA and 5 days, with rigorous follow-

${ }^{*}$ Correspondence to: Enora PARC, Hospital of the Eastern Paris Hospital Group, site Jossigny, France, Tel: 06296806 03, E-mail : enora.parc@gmail.com Key words: postterm pregnancy, antenatal follow-up, induction of labour

Received: September 10, 2019; Accepted: September 23, 2019; Published: September 26, 2019 
PARC E (2019) Before-after study comparing systematic labour induction at 41 weeks of amenorrhea and 3 days versus 41 weeks of amenorrhea and 5 days in a maternity ward in France in 2017-2018

up in both cases, and evaluating different maternal and fetal morbidity criteria, in order to improve our practice.

\section{Materials and method}

We performed a retrospective descriptive observational study, "before-after" type. Our study took place in the $2 \mathrm{~b}$ maternity hospital of the Eastern Paris Hospital Group, Jossigny, France. The first study period was from September to November 2017, when maternity protocol was a systematic induction at $41 \mathrm{WA}+3$ days. The second period ran from March to May 2018, a few months after the maternity protocol was modified and indicated a systematic induction at $41 \mathrm{WA}$ +5 days.

We included all patients who gave birth on both periods after 41 WA +3 days. The only exclusion criterion was spontaneous labour of patients before $41 \mathrm{WA}+3$ days.

For the first group, the protocol was as follows: all patients were systematically seen at $41 \mathrm{WA}$ and $41 \mathrm{WA}+2$ days. They benefited from a monitoring of the foetal heart rate and tocometry, a clinical examination of the cervix, an ultrasound to check the quantity of amniotic fluid. In case of strictly normal examination, and if they did not start spontaneously labor, the patients were asked to come back at $41 \mathrm{WA}+3$ days to induce labour according to their cervical conditions. For the second group, the protocol was identical at $41 \mathrm{WA}, 41 \mathrm{WA}+2$ days and $41 \mathrm{WA}+4$ days. Then patients were systematically induced at $41 \mathrm{WA}+5$ days if not delivered and if the various examinations were always strictly normal.

Our induction protocol depended on the patient BISHOP score, parity, and having a scarred uterus or not (Appendix 1). For a primiparous with a score $<6$, we used prostaglandins (by tampon if score $<3$, by gel if score of 4 or 5 ). The $\operatorname{Cook} ®$ cervical ripening balloon was used in case of scared uterus or large multiparity and score $<6$. Finally, for a score $>$ or $=$ to 6 after $41 \mathrm{WA}$, patients were systematically induced by artificial rupture of the membranes and perfusion of oxytocin.

The primary endpoint of the study was a composite criterion of maternal and foetal morbidity, including delivery characteristics, caesarean section rate, and neonatal status. To carry out this study, we used the data from our patients' computerized records. Patients were characterized by age, parity, significant past medical history, and their current pregnancy's complications.

We detailed the modes of induction as well as their reasons: post term pregnancy only, decreasing of perceived foetal active movements, oligoamnios, spontaneous rupture of membranes with a positive vaginal sample (Streptococcus B or Escherichia Coli K1) abnormal foetal heart rate. Vaginal deliveries were distinguished from caesareans, which indications were specified. Perineal status, estimation of blood loss, and the colour of the amniotic fluid at expulsion were described. Concerning the newborn, neonatal status was specified by its birth weight, APGAR score, neonatal $\mathrm{pH}$, and potential transfer to neonatal intensive care unit.

We compared characteristics of the two populations, "before" and "after". Analysis was performed by Chi 2 tests for categorical variables, and Kruskall-Wallis tests for medians of continuous variables. We did not have any missing data in this study. All analyzes were done on the software STATA Version 13.

\section{Results}

We included 64 patients in the "before" group and 54 patients in the "after" group. Regarding maternal characteristics (Table 1), the two groups were comparable: There was no significant difference, especially concerning parity. Two of the patients included had an imprecise pregnancy term because these pregnancies were dated after 13 WA and 6 days. Significant past medical history was maternal pathologies that could have an impact on the current pregnancy: hypothyroidism and coagulation disorders. We grouped these different pathologies under the same entity, seeing that the majority of included patients had no particular medical nor surgical history. Similarly, there was no significant difference in the pregnancies' complications between the two groups.

Table 2 shows that we found a higher induction rate in the "before" group (systematic induction at $41 \mathrm{WA}+3$ days) compared to the

Table 1. Characteristics of the two populations "before" / "after"

\begin{tabular}{|c|c|c|c|c|c|c|}
\hline \multirow[t]{2}{*}{ VARIABLES } & \multirow[b]{2}{*}{ categorical } & \multicolumn{2}{|c|}{ BEFORE } & \multicolumn{2}{|c|}{ AFTER } & \multirow{3}{*}{$\mathbf{p}$} \\
\hline & & $\mathbf{N}$ & $\%$ & $\mathbf{N}$ & $\%$ & \\
\hline & continuous & median & $(\min -\max )$ & median & $(\min -\max )$ & \\
\hline Age & & 30,5 & $(18-42)$ & 31,5 & $(20-41)$ & 0,49 \\
\hline \multirow{3}{*}{ Parity } & Primipara & 38 & 59,4 & 21 & 38,9 & \multirow{3}{*}{0,06} \\
\hline & Multipara with healthy uterus & 20 & 31,3 & 22 & 40,7 & \\
\hline & Multipara with scarred uterus & 6 & 9,4 & 11 & 20,4 & \\
\hline Significant pathologies & & 7 & 10,9 & 10 & 18,5 & 0,24 \\
\hline Non-precise term & & 2 & 3,1 & 0 & 0,0 & 0,19 \\
\hline \multirow{7}{*}{ Progress of pregnancy } & Normal pregnancy & 49 & 76,6 & 45 & 83,3 & \multirow{7}{*}{0,58} \\
\hline & IVF* pregnancy & 2 & 3,1 & 1 & 1,9 & \\
\hline & Balanced gestational diabetes (without macrosomia) & 6 & 9,4 & 6 & 11,1 & \\
\hline & Suspicion of foetal macrosomia (without gestationel diabetes) & 1 & 1,6 & 1 & 1,9 & \\
\hline & Breech infant & 3 & 4,7 & 0 & 0,0 & \\
\hline & Small for gestational age & 2 & 3,1 & 0 & 0,0 & \\
\hline & Thrombocytopenia & 1 & 1,6 & 1 & 1,9 & \\
\hline
\end{tabular}


PARC E (2019) Before-after study comparing systematic labour induction at 41 weeks of amenorrhea and 3 days versus 41 weeks of amenorrhea and 5 days in a maternity ward in France in 2017-2018

"after" group (systematic induction at $41 \mathrm{WA}+5$ days). By extension, spontaneous labour rate is significantly higher in the "after" group.

Two patients had caesarean sections before labour in the "after" group: breech foetus and suspicion of macrosomia without gestational diabetes, both in the context of a scarred uterus. The reason for the majority of inductions was the post term context only, whether at 41 $\mathrm{WA}+3$ days or $41 \mathrm{WA}+5$ days according to the group. There was a significant difference regarding the reasons for induction: more inductions only for post term in the "before" group, and more induction for other reasons (oligoamnios, decreased active foetal movement, abnormal foetal heart rate and premature rupture of the membranes) in the "after" group. Due to our study small size, it is difficult to interpret individually the differences in the induction reasons between the two groups. At last there was no difference between different induction methods used in the two groups.

There was no significant difference in the delivery modes between the two groups, as shown in Table 3, whether vaginal delivery (spontaneous or instrumental) or caesarean section during labour ( $\mathrm{p}=$ 0.61). For caesarean sections, we compared caesarean delivery rates by combining caesarean sections that took place before labour and those performed during labor, but there was no significant difference either ( $\mathrm{p}$ $=0.12$ ). There was also no significant difference between the caesarean sections indications during labour between the two groups $(\mathrm{p}=0.36)$.

For patients who had vaginal deliveries, there was no difference in perineal lesions $(p=0.06)$, estimated total blood loss $(p=0.76)$, or color of amniotic fluid at expulsion $(\mathrm{p}=0.28)$.

Median birth weight was comparable between the two groups $(\mathrm{p}=$ 0.57 ) (Table 4$)$, as was neonatal APGAR score ( $p=0.92,0.95$ and 0.61 for APGAR score at 1 minute, 3 minutes and 5 minutes of life). In both groups, there was one child with a neonatal $\mathrm{pH}$ of less than 7.10, and there was no newborn with a $\mathrm{pH}$ lower than 7.00. It should be noted that there were three transfers in neonatology in the "before" group: two for suspicion of maternofoetal infection and one for respiratory distress, and there were none in the "after" group, but without significative difference $(\mathrm{p}=0.11)$.

\section{Discussion}

In total, caesarean section rate was not increased and neonatal status comparable in the "after" group induced at 41 WA +5 days, compared to the "before" group induced at $41 \mathrm{WA}+3$ days.

Significant differences concerned rates and indications of induction between the two groups. At $41 \mathrm{WA}+5$ days, rate of spontaneous labor is greater, and decreased of systematic induction between $41 \mathrm{WA}+3$ days and $41 \mathrm{WA}+5$ days clearly shows that maternofoetal surveillance has been systematic in our unit, given that a labour induction was indicated if there was any anomaly during the surveillance, without taking further risk for the mother or the child.

These results are consistent with some other studies on this subject, such as the observational study carried out in the Pellegrin Hospital of Bordeaux (level 3 maternity unit), over a period going from January 2005 to March 2011 [14]. The authors compared a medical induction of labor between $41 \mathrm{WA}+2$ days and $41 \mathrm{WA}+4$ days. Patients were monitored from 41 WA onwards following a procedure similar to ours. The induction was only performed using prostaglandins. The primary endpoint was the caesarean section rate, and no significant difference was found in either group. Secondary criteria on neonatal status were comparable in both groups. However, they found a higher rate of foetal heart rate abnormalities during labour and instrumental extractions in the $41 \mathrm{WA}+4$ days group. This result was related to a higher rate of nulliparas in this group, which caused an interpretation bias.

Our study had several limitations: A power insufficiency due to the size of the study, the fact that it was unicentric, over a limited period of time. Moreover, the study compared two populations with a very close

Table 2. Characteristics of labour

\begin{tabular}{|c|c|c|c|c|c|c|}
\hline \multirow[t]{3}{*}{ VARIABLES } & \multirow{3}{*}{$\begin{array}{l}\text { categorical } \\
\text { continuous }\end{array}$} & \multicolumn{2}{|c|}{ BEFORE } & \multicolumn{2}{|c|}{ AFTER } & \multirow{3}{*}{$\mathbf{p}$} \\
\hline & & \multirow{2}{*}{$\begin{array}{c}\mathrm{N} \\
\text { median }\end{array}$} & \multirow{2}{*}{$\begin{array}{c}\% \\
(\min -\max )\end{array}$} & \multirow{2}{*}{$\begin{array}{c}\mathbf{N} \\
\text { median }\end{array}$} & \multirow{2}{*}{$\begin{array}{c}\% \\
(\min -\max )\end{array}$} & \\
\hline & & & & & & \\
\hline \multirow{5}{*}{$\begin{array}{c}\text { Delivery term } \\
\text { (in weeks of } \\
\text { amenorrhea + days) }\end{array}$} & $41+3$ & 28 & 43,8 & 13 & 24,1 & \multirow{5}{*}{$<0,01$} \\
\hline & $41+4$ & 24 & 37,5 & 11 & 20,4 & \\
\hline & $41+5$ & 11 & 17,2 & 12 & 22,2 & \\
\hline & $41+6$ & 1 & 1,6 & 17 & 31,5 & \\
\hline & $42+0$ & 0 & 0,0 & 1 & 1,9 & \\
\hline \multirow{3}{*}{ Labour } & Spontaneous & 1 & 1,6 & 16 & 29,6 & \multirow{3}{*}{$<\mathbf{0 , 0 1}$} \\
\hline & Induction of labour & 63 & 98,4 & 36 & 66,7 & \\
\hline & Caesarean before labour & 0 & 0,0 & 2 & 3,7 & \\
\hline \multirow{7}{*}{ Induction's indication } & & $\mathrm{N}=63$ & & $\mathrm{~N}=36$ & & \\
\hline & Post term only & 56 & 88,9 & 23 & 63,9 & \multirow{6}{*}{$\mathbf{0 , 0 1}$} \\
\hline & Oligoamnios & 2 & 3,2 & 3 & 8,3 & \\
\hline & Decreased foetal active movements & 0 & 0,0 & 1 & 2,8 & \\
\hline & Foetal heart rate abnormalities & 1 & 1,6 & 7 & 19,4 & \\
\hline & Premature rupture of membranes & 3 & 1,8 & 2 & 5,6 & \\
\hline & Thrombocytopenia & 1 & 1,6 & 0 & 0,0 & \\
\hline \multirow{4}{*}{ Induction procedure } & Prostaglandin vaginal tampon & 42 & 66,7 & 23 & 63,9 & \multirow{4}{*}{0,58} \\
\hline & Prostaglandin vaginal gel & 4 & 6,4 & 2 & 5,6 & \\
\hline & Cook's balloon & 5 & 7,9 & 6 & 16,7 & \\
\hline & Oxytocin & 12 & 19,1 & 5 & 13,9 & \\
\hline
\end{tabular}


PARC E (2019) Before-after study comparing systematic labour induction at 41 weeks of amenorrhea and 3 days versus 41 weeks of amenorrhea and 5 days in a maternity ward in France in 2017-2018

Table 3. Delivery characteristics

\begin{tabular}{|c|c|c|c|c|c|c|}
\hline \multirow[t]{3}{*}{ VARIABLES } & \multirow[b]{2}{*}{ categorical } & \multicolumn{2}{|c|}{ BEFORE } & \multicolumn{2}{|c|}{ AFTER } & \multirow{3}{*}{$\mathbf{p}$} \\
\hline & & \multirow{2}{*}{$\frac{\mathrm{N}}{\text { median }}$} & \multirow{2}{*}{$\frac{\%}{(\min -\max )}$} & \multirow{2}{*}{$\begin{array}{c}\mathrm{N} \\
\text { median }\end{array}$} & \multirow{2}{*}{$\begin{array}{c}\% \\
(\min -\max )\end{array}$} & \\
\hline & continuous & & & & & \\
\hline \multirow{3}{*}{ Delivery mode } & Spontaneous vaginal delivery & 32 & 50,0 & 27 & 51,9 & \\
\hline & Instrumental delivery & 10 & 15,6 & 11 & 21,2 & 0,61 \\
\hline & Caesarean section during labor & 22 & 34,4 & 14 & 26,9 & \\
\hline \multirow{4}{*}{$\begin{array}{l}\text { Indication in case } \\
\text { of caesarean during } \\
\text { labour }\end{array}$} & & $\mathrm{N}=22$ & & $\mathrm{~N}=14$ & & \\
\hline & Failure of induction & 4 & 18,2 & 5 & 35,7 & \\
\hline & Stagnation of dilatation & 11 & 50,0 & 4 & 28,6 & 0,36 \\
\hline & Foetal heart rate abnormalities & 7 & 31,8 & 5 & 35,7 & \\
\hline \multirow{5}{*}{$\begin{array}{c}\text { Perineum status } \\
\text { (in vaginal deliveries) }\end{array}$} & Intact & 16 & 38,1 & 8 & 21,1 & \\
\hline & Episiotomy & 7 & 16,7 & 5 & 13,2 & \\
\hline & Simple tear & 18 & 42,9 & 21 & 55,3 & 0,06 \\
\hline & Complete uncomplicated perineum & 0 & 0,0 & 4 & 10,5 & \\
\hline & Complete and complicated perineum & 1 & 2,4 & 0 & 0,0 & \\
\hline \multirow{3}{*}{$\begin{array}{l}\text { Estimation of blood } \\
\text { loss }\end{array}$} & Physiological $(<500 \mathrm{cc})$ & 59 & 92,2 & 49 & 90,7 & \\
\hline & Non-severe haemorrhage $(<1000 \mathrm{cc})$ & 4 & 6,3 & 3 & 5,6 & 0,76 \\
\hline & Severe haemorrhage $(>1000 \mathrm{cc})$ & 1 & 1,6 & 2 & 3,7 & \\
\hline \multirow{3}{*}{$\begin{array}{l}\text { Color of amniotic } \\
\text { fluid at expulsion }\end{array}$} & Clear & 49 & 76,6 & 39 & 72,2 & \\
\hline & Tinted & 11 & 17,2 & 7 & 13,0 & 0,28 \\
\hline & Meconial & 4 & 6,3 & 8 & 14,8 & \\
\hline
\end{tabular}

Table 4. Neonatal characteristics

\begin{tabular}{|c|c|c|c|c|c|c|}
\hline \multirow[t]{3}{*}{ VARIABLES } & \multirow[b]{2}{*}{ categorical } & \multicolumn{2}{|c|}{ BEFORE } & \multicolumn{2}{|c|}{ AFTER } & \multirow{3}{*}{$\mathbf{p}$} \\
\hline & & $\mathbf{N}$ & $\%$ & $\mathbf{N}$ & $\%$ & \\
\hline & continuous & median & $(\min -\max )$ & median & $(\min -\max )$ & \\
\hline Birth weight & & 3590 & $(2245-4750)$ & 3610 & $(2885-4965)$ & 0,57 \\
\hline \multirow{3}{*}{ APGAR score } & 1 minute of life & 10 & $(1-10)$ & 10 & $(2-10)$ & 0,92 \\
\hline & 3 minutes of life & 10 & $(7-10)$ & 10 & $(5-10)$ & 0,95 \\
\hline & 5 minutes of life & 10 & $(8-10)$ & 10 & $(8-10)$ & 0,61 \\
\hline \multirow{4}{*}{ Neonatal cord $\mathrm{pH}$} & $\mathrm{pH}>=7,20$ & 47 & 81,0 & 44 & 86,3 & \multirow{4}{*}{0,72} \\
\hline & $7,10<=\mathrm{pH}<7,20$ & 10 & 17,2 & 6 & 11,8 & \\
\hline & $7,00<=\mathrm{pH}<7,10$ & 1 & 1,7 & 1 & 2,0 & \\
\hline & $\mathrm{pH}<7,00$ & 0 & 0,0 & 0 & 0,0 & \\
\hline Shoulders' dystocia & & 1 & 1,6 & 3 & 5,6 & 0,23 \\
\hline \multicolumn{2}{|c|}{ Transfer to Neonatal Intensive Care Unit } & 3 & 4,7 & 0 & 0,0 & 0,11 \\
\hline
\end{tabular}

term: there is an uncertainty of about 3 days of first trimester ultrasound to determine the exact beginning of a pregnancy, which may explain the absence of significant difference between the two groups on endpoints. The strength of this study is that it provides additional arguments for an induction at $41 \mathrm{WA}+5$, which is not currently the consensus in France or other countries.

In the United Kingdom, official medical societies recommend close monitoring from $41 \mathrm{WA}$, with possible induction as soon as local conditions are favourable. Surveillance can be extended after $42 \mathrm{SA}$, unlike France [15].

The surveillance and induction procedures are identical in Canada, with additional offering of membrane detachment from 37 WA onwards [16].

The results of this study however, confirmed implementation of this new protocol in our maternity unit. This protocol is in line with the latest recommendations for the clinical practice of post term pregnancies of 2011 [1]. To generalize this protocol, it would be interesting to carry out a randomized study on a larger scale.

\section{Conflict of interest}

The authors declare that they have no conflict of interest.

\section{References}

1. CPR (Recommendations for Clinical Practice) Prolonged Pregnancy and Post term December 2011

2. Blondel B, Supernant K, Du Mazaubrun C, Bréart G. Trends in perinatal health in metropolitan France between 1995 and 2003: results from the National Perinatal Surveys. J Gynecol Obstet Biol Reprod (Paris) 2006 june.

3. Blondel B, Coulm B, Bonnet C, Bonnet F, Goffinet F, et al. Trends in perinatal health in metropolitan France from 1995 to 2016: Results from the French National Perinatal Survey National. Coordination Group of the National Survey.

4. Olesen AW, Westergaard JG, Olsen J (2003) Perinatal and maternal complications related to postterm delivery: a national register-based study, 1978-1993. Am J Obstet Gynecol 189: 222-227. [Crossref]

5. Cheng YW, Nicholson JM, Nakagawa S, Bruckner TA, Washington AE, et al. (2008) Perinatal outcomes in low-risk term pregnancies: do they differ by week of gestation? Am J Obstet Gynecol 199: 370.e1-7. [Crossref]

6. Caughey AB, Musci TJ (2004) Complications of term pregnancies beyond 37 weeks of gestation. Obstet Gynecol 103: 57-62. [Crossref] 
PARC E (2019) Before-after study comparing systematic labour induction at 41 weeks of amenorrhea and 3 days versus 41 weeks of amenorrhea and 5 days in a maternity ward in France in 2017-2018

7. Caughey AB, Stotland NE, Washington AE, Escobar GJ (2007) Maternal and obstetric complications of pregnancy are associated with increasing gestational age at term. $\mathrm{Am}$ J Obstet Gynecol 196: 155.e1-6. [Crossref]

8. Gülmezoglu AM, Crowther CA, Middleton P (2006) Induction of labour for improving birth outcomes for women at or beyond term. Cochrane Database Syst Rev 13: CD004945. [Crossref]

9. Heimstad R, Skogvoll E, Mattsson LA, Johansen OJ, Eik-Nes SH, et al. (2007) Induction of labor or serial antenatal fetal monitoring in postterm pregnancy: a randomized controlled trial. Obstet Gynecol 109: 609-617. [Crossref]

10. Alexander JM, McIntire DD, Leveno KJ (2000) Forty weeks and beyond: pregnancy outcomes by week of gestation. Obstet Gynecol 96: 291-294. [Crossref]
11. Ehrenstein V, Pedersen L, Holsteen V, Larsen H, Rothman KJ, et al. (2007) Postterm delivery and risk for epilepsy in childhood. Pediatrics 119: e554-e561. [Crossref]

12. Hilder L, Costeloe K, Thilaganathan B. Prolonged pregnancy: evaluating gestationspecific risks of fetal and infant mortality. Br J Obstet Gynaecol 105: 169-173. [Crossref]

13. Artificial induction of labor from 37 weeks of amenorrhea, professional recommendations. HAS 2008.

14. Syngof revue $\mathrm{N}^{\circ} 93$, june 2013, prolonged pregnancy and post term.

15. Induction of labour, National Collaborating Centre for Women's and Children's Health, Commissioned by the National Institute for Health and Clinical Excellence, July 2008.

16. Guidelines for the management of pregnancy at $41+0$ to $42+0$ weeks (2008) $\mathrm{J}$ Obstet Gynaecol.

Copyright: (C2019 PARC E. This is an open-access article distributed under the terms of the Creative Commons Attribution License, which permits unrestricted use, distribution, and reproduction in any medium, provided the original author and source are credited. 